\title{
Monopolar pulsar spin-down
}

\author{
C. Alvarez and A. Carramiñana
}

\author{
Instituto Nacional de Astrofísica, Óptica y Electrónica, Luis Enrique Erro 1, Tonantzintla, Puebla 72840, México \\ e-mail: calvarez@inaoep.mx; alberto@inaoep.mx
}

Received 16 January 2003 / Accepted 14 October 2003

\begin{abstract}
A multipole spin-down equation based on a monopolar term is derived from the general expression $\dot{v}=-f(v, t)$ and used to study pulsar evolution. We show that the time-independent version of such equation cannot reproduce the observed properties of pulsars and conclude that there is no equation of the form $\dot{v}=-f(v)$ consistent with the $P-\dot{P}$ diagram and braking index measurements. We explore the time-dependent model under the hypothesis of decaying magnetic fields, showing that an inverse linear decay gives reasonable evolutionary trajectories. This model distinguishes the evolution of Vela from that of the other three young pulsars considered. We discuss the origin of the monopolar term, which cannot be attributed to radiative processes, pointing to the importance of particle acceleration and/or mass loss processes in the dynamical evolution of pulsars.
\end{abstract}

Key words. stars: neutron - stars: pulsars: general

\section{Introduction}

Pulsars are accepted to be rapidly rotating and highly magnetized neutron stars powered by the loss of rotational kinetic energy. Yet, the mechanisms through which pulsars lose their rotational energy are not very well understood. The discovery of pulsars was preluded by the rotating magnetic dipole model of Pacini (1967), which predicted a spin-down relation $\dot{v} \propto v^{3}$, where $v$ is the rotational frequency. Pulsar spin-down has then been studied through the power-law differential equation,

$\dot{v}=-k v^{n}$,

where $k$ is a constant and $n$ is called the "braking index", determined by the physical mechanisms spinning-down the star. For a pure magnetic dipole $n$ is equal to 3 (Gold 1968; Pacini 1968), while a pure gravitational or electromagnetic quadrupole one has $n=5$ (Ostriker \& Gunn 1969; Ferrari \& Ruffini 1969). The expansion of the Larmor equation into electromagnetic moments higher than dipolar gives $n>3$. Departures from these basic models have been studied. For example, the deformation of magnetic field lines by corotation with the magnetosphere might produce $1 \leq n \leq 3$ (Manchester \& Taylor 1977), pulsar winds $n<3$ (Blandford \& Romani 1988; Manchester et al. 1985), while magnetic field decay (Chanmugam \& Sang 1989) and the alignment between rotation and magnetic axis tend to give $n>3$ (Goldreich 1970). Physical processes such as phase transitions in the interior of the fast rotating neutron stars can produce changes in the moment of inertia $I$ and led also to deviations from $n=3$ even if the pulsar is spinning-down by pure dipolar radiation (Chubarian et al. 2000; Glendenning et al. 1997). However, these phase transitions are significant only for

Send offprint requests to: C. Alvarez, e-mail: calvarez@inaoep.mx very fast pulsars, with periods $P<1.5 \mathrm{~ms}$. For pulsars with periods $P \gtrsim 3 \mathrm{~ms}$, these changes in the moment of inertia can be neglected. But while physical mechanisms to produce $n<3$ have been proposed, the reconciliation of pulsar evolution with $n<3$ remains to be studied. This question, addressed to the case of young classical pulsars, is one of the main objectives of this work.

The braking index $n$ is frequently defined in terms of observational quantities, with a definition generalized to a second braking index, $m$ :

$n \equiv \frac{\ddot{v} v}{\dot{v}^{2}}, \quad m \equiv \frac{\dddot{v} v^{2}}{\dot{v}^{3}}$.

Accurate measurement of both braking indices requires longterm timing measurements, a difficult task due to the existence of glitches and timing noise in young pulsars, affecting the measurement of $\ddot{v}$ and $\ddot{v}$. Out of more than 1300 detected pulsars, only a handfull have reliable braking index measurements (Table 1). Still, measurements continue to be made and objects like PSR J1119-6127 and PSR J1846-0258 might probably be recent additions to the list (Camilo et al. 2000; Mereghetti et al. 2002). The second braking index $m$ is even more difficult to measure and has only been estimated for PSR B1509-58 (Kaspi et al. 1994) and the Crab pulsar (Lyne et al. 1988) (Table 1). It is well-known that measured values of $n$ and $m$ are below, but close, to those of a magnetic dipole in vacuum ( $n=3$ and $m=15)$.

Pulsar evolution is mostly studied through the $P-\dot{P}$ diagram, which shows a sparse distribution of pulsars clustered roughly at $\log P \sim-0.3$ and $\log \dot{P} \sim-15$. In principle it should be possible to link the bulk of the pulsar population in the $P-\dot{P}$ diagram with the younger pulsars through evolutionary 
Table 1. Timing parameters and stationary multipole model fitting parameters for the four young pulsars selected.

\begin{tabular}{lcccc}
\hline \hline Pulsar & $\mathrm{Crab}^{(1)}$ & $1509-58^{(2)}$ & $0540-69^{(3)}$ & Vela $^{(4)}$ \\
\hline$P(\mathrm{~ms})$ & 33.5 & 150.9 & 50.3 & 89.3 \\
$t_{\text {dyn }}(\mathrm{yr})$ & 1258 & 1553 & 1664 & 11300 \\
$n$ & 2.509 & 2.837 & 2.01 & 1.4 \\
& \pm 0.005 & \pm 0.001 & \pm 0.02 & \pm 0.2 \\
$m$ & 10.23 & 14.5 & $\ldots$ & $\ldots$ \\
& \pm 0.03 & \pm 3.6 & $\ldots$ & $\ldots$ \\
\hline$g\left(\mathrm{~Hz}^{-3}\right)$ & $3.5 \times 10^{-19}$ & $6.2 \times 10^{-16}$ & $6.6 \times 10^{-18}$ & $3.8 \times 10^{-18}$ \\
$r\left(\mathrm{~Hz}^{-1}\right)$ & $1.0 \times 10^{-14}$ & $1.6 \times 10^{-13}$ & $7.1 \times 10^{-15}$ & $1.3 \times 10^{-15}$ \\
$s(\mathrm{~Hz})$ & $3.4 \times 10^{-12}$ & $2.0 \times 10^{-12}$ & $5.7 \times 10^{-12}$ & $1.2 \times 10^{-12}$ \\
\hline$P_{\text {birth }}$ & 9.9 & $14.2^{b}$ & 17.6 & 50 \\
$(\mathrm{~ms})^{a}$ & \multicolumn{5}{c}{} \\
\hline
\end{tabular}

${ }^{a} P_{\text {birth }}$ defined as the period one dynamical time ago.

${ }^{b}$ Integration is stopped at $t_{\mathrm{dyn}}=1$ year.

(1) Lyne et al. (1988), (2) Kaspi et al. (1994), (3) Manchester \& Peterson (1989), (4) Lyne et al. (1996).

tracks consistent with a general spin-down equation. However, neither the magnetic dipole model or a constant braking index equation can link these groups and it has been argued that pulsars might be born with small period derivatives, in a region of the $P-\dot{P}$ diagram where no pulsar detection has been reported (Camilo 1996). Alternatively, magnetic dipole moments might decay during the pulsar life (Camilo 1996; Tauris \& Konar 2001). On these lines, Colpi et al. (2000) studied the distribution and evolution in the $P-\dot{P}$ diagram of anomalous X-ray pulsars (AXPs), considering dipolar spin-down of magnetars. They conclude that magnetic field decay is required to account for the observed distribution and X-ray luminosities.

We propose here a spin-down model derived from the very general spin-down law $\dot{v}=-f(v, t)$. Although the derivation is empirical, the model accomodates the usual spin-down mechanisms, namely magnetic dipole radiation and gravitational radiation. In addition, a monopolar term is introduced, aiming to reconcile braking indices with trajectories in the $P-\dot{P}$ diagram. We calculate evolutionary trajectories for PSR B1509-58, PSR B0540-69, the Vela pulsar (PSR B0833-45) and the Crab pulsar (PSR B0531+21). The model is presented in Sect. 2, while in Sect. 3 we apply the model to the four pulsars just mentioned, assuming time-independent spin-down mechanisms. In Sect. 4 we study the model considering timedependent spin-down mechanisms. In Sect. 5, a discussion of possible physical mechanisms related to the spin-down terms is given, to lead to the concluding Sect. 6.

\section{The model}

The basic assumption of this work is that the frequency derivative $\dot{v}$ can be described as a function of frequency $v$ and time $t$ only:

$\dot{v}=-f(v, t)$.
We then assume the following properties:

1. $f>0$ for all $(v, t)$, as $f$ describes energy and angular momentum losses.

2. $f$ is a continuous function of time. This requires that the cumulative effect of glitches on evolutionary paths in the $P-\dot{P}$ diagram can be neglected.

3. $f$ is an antisymmetric function, $f(-v, t)=-f(v, t)$. This is equivalent to ascribe a sign to the frequency $v$ and its derivative $\dot{v}: v$ and $\dot{v}$ have the same signs when the pulsar is spinning-up and opposite signs when the pulsar spinsdown. This condition will be further discussed below.

The model consists then of a simple Taylor expansion of $f$, restricted to the three lowest order terms:

$\dot{v}=-s(t) v-r(t) v^{3}-g(t) v^{5}$.

Higher order terms can be neglected for the frequencies measured in known isolated pulsars ${ }^{1}$. A variable moment of inertia, $\dot{I} \neq 0$, would introduce an extra positive monopole term $\dot{I} / 2 I$ that can be incorporated in $s(t)$. This would affect only very fast pulsars and we will consider $I$ constant in here. Equation (4) represents a very general spin-down model for isolated pulsars. Beyond its simple empirical derivation, it can represent the loss of rotational energy through physical processes described by standard electromagnetic and gravitational radiation multipolar terms, together with the ad hoc "monopolar" term. We will argue later that this term has to be associated with other type of processes, like particle acceleration or pulsar winds. We underline that if this model cannot be used to interpret the $P-\dot{P}$ diagram, then there is no analytical equation of the form given by Eq. (3), consistent with the required conditions, able to reconcile braking indices with pulsar evolution.

\section{The stationary multipole spin-down model}

Leaving momentarily aside the discussion of the origin of the different terms, we consider now the model given by Eq. (4) when the coefficients $g, r$ and $s$ are constant, following Alvarez \& Carramiñana (1998). These coefficients can be calculated if the frequency and both braking indices are known. Taking the first and second derivative of Eq. (4) and using Eqs. (2), one arrives to a matrix equation which can be inverted to give:

$$
\left(\begin{array}{c}
g v^{5} /(-\dot{v}) \\
r v^{3} /(-\dot{v}) \\
s v /(-\dot{v})
\end{array}\right)=\frac{1}{8}\left(\begin{array}{rrr}
3 & -3 & 1 \\
-10 & 10 & -2 \\
15 & -7 & 1
\end{array}\right)\left(\begin{array}{c}
1 \\
n \\
m-n^{2}
\end{array}\right)
$$

The factors $g v^{5} /(-\dot{v}), r v^{3} /(-\dot{v})$ and $s v /(-\dot{v})$ represent the fractions of the total energy loss contained in the quadrupole, dipole and monopole terms respectively. Constraining each term to be between 0 and 1 leads to the bounds on their values as function of $n$ represented in Fig. 1. The value of $m$ is restricted to the range

$\operatorname{Max}\left(n^{2}+3 n-3 ; n^{2}+7 n-15\right) \leq m \leq n^{2}+5 n-5$,

while $1 \leq n \leq 5$. The minimum and maximum allowable values for $m$ are 1 and 45, which happen for $n=1$ and $n=5$

\footnotetext{
${ }^{1}$ Binary and millisecond pulsars are not considered here.
} 

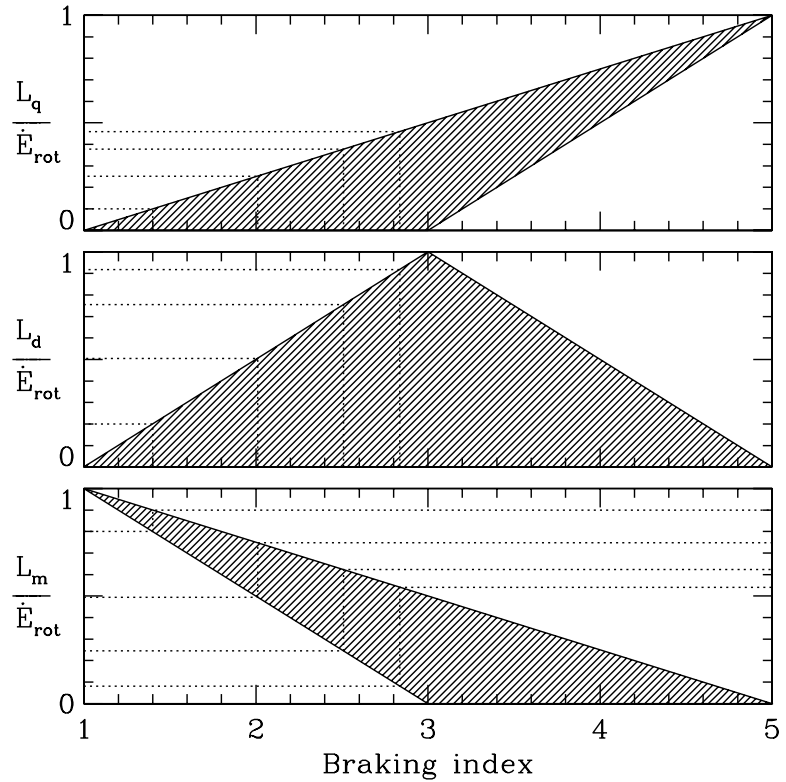

Fig. 1. $L / \dot{E}_{\text {rot }}$ ratios for the quadrupole (upper panel), dipole (middle panel) and monopole (lower panel) terms. Only values inside the shaded areas are allowed by the stationary version of the model. When $n \rightarrow 1$ and $n \rightarrow 5$ the rotational losses are due only to the monopole or quadrupole term respectively. The observed braking indices give upper and lower limits for the different $L / \dot{E}_{\text {rot }}$ ratios, as indicated by the dotted lines for Vela, PSR B0540-69, the Crab and PSR B1509-58 (from left to right in the horizontal axis).

respectively. Accordingly, pulsars must be in a restricted region of the $(n, m)$ plane defined by Eq. (6), and shown in Fig. 2. Spin-down evolution is initially dominated by the quadrupole term $(n \rightarrow 5)$, afterwards by the dipole term $(n \rightarrow 3)$ and finally by the monopole term $(n \rightarrow 1)$. Under this model the braking index itself would be a rough qualitative age indicator.

From the known timing parameters, $v, \dot{v}, n$ and $m$, one determines $\{g, r, s\}$ uniquely, from the matrix relation (5), with no degree of freedom. This could only be done for PSR B1509-58, while for the Crab, PSR B0540-69 and Vela pulsars we assumed $m$ within the constraints of the model. Evolutionary tracks were calculated for these pulsars, integrating Eq. (4) backwards one dynamical time and forward to a point where the monopole term dominates the spin-down evolution (Fig. 3). The results for each of the four pulsars can be summarized as follows:

- the second braking index of the Crab pulsar, $m=10.23$, is below the minimum value allowed by the model $(m \geq 10.82)$ and in order to compute evolutionary trajectories we used $m=11$. This value of $m$ is consistent with the initial period calculated in other models for this pulsar (Mereghetti et al. 2002; Glendenning 1996). The Crab is the only pulsar studied here with the precise age known, allowing some specific comparisons. Integrating back to its actual birth (949 years ago) one finds an initial period $\$ 18 \mathrm{~ms}$ (Fig. 4). If we integrate back one dynamical time (1258 years) from now, we get an initial period of $10 \mathrm{~ms}$, lower but within a factor of two of the period at birth.

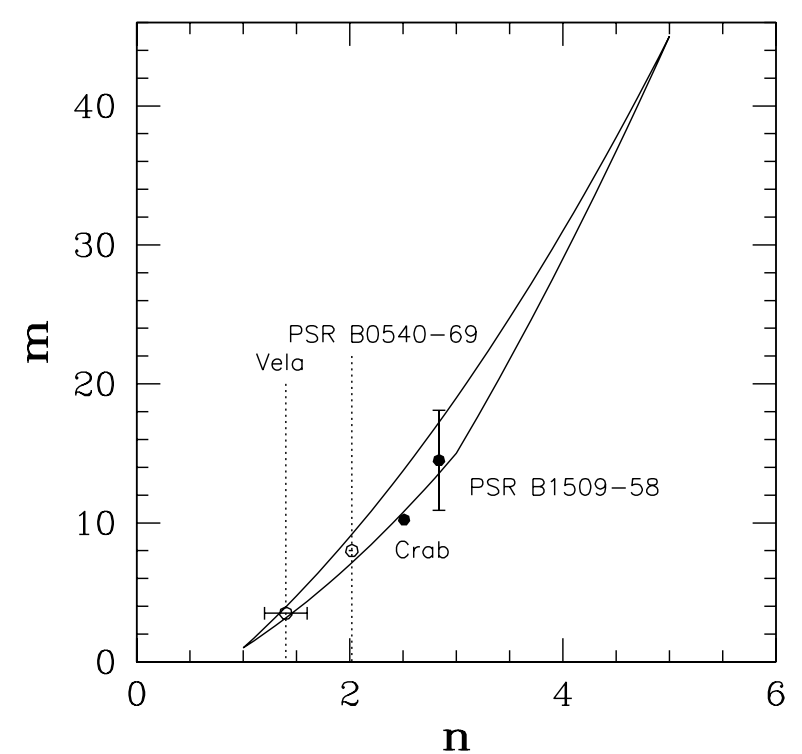

Fig. 2. Allowed values of the braking indices $n$ and $m$. According to the stationary version of the model, pulsars evolve from the upperright to the lower-left, increasing their braking indices, but keeping them inside the triangle-like region. Both indices have been measured only for PSR B1509-58, which lies inside the allowed region, and the Crab pulsar, located slightly outside the allowed region. For Vela and PSR B0540-69 the value of $m$ has not been measured and we have assumed $m$ to be in the middle of the allowed range (open circles). Vertical dot lines indicate the value of $n$ of these pulsars. Errors of $n$ for the Crab and PSR B1509-58 are smaller than the dots.

- for PSR B1509-58 the measured values of $m$ and $n$ are consistent with the model. However, the integration of Eq. (4) could not be carried out one entire dynamical time backwards, as $\int \mathrm{d} t$ converges at about 1350 years, i.e., before the present dynamical time (1553 years). Integrating back to a dynamical age of 1 year, we obtain a putative "initial period" of about $14 \mathrm{~ms}$ and a $\dot{P}$ two orders of magnitude higher than for any of the other three pulsars. This pulsar seems to have been formed in a particular region of the $P-\dot{P}$ diagram. Its quadrupolar parameter $g$ is at least two orders of magnitude higher that for the other pulsars. An initial phase of strong gravitational spin-down would naturally lead to the high $P$ and $\dot{P}$ observed in this pulsar.

- PSR B0540-69: its second braking index is still unknown so we took $m=8.0$, in the middle of the range of allowed values. As the known timing parameters of PSR B0540-69 are similar to those of the Crab, albeit a somewhat longer period, their evolutionary paths are similar, with an initial $P-\dot{P}$ position of PSR B0540-69 relatively close to that of the Crab.

- Vela pulsar: the frequent glitches of Vela prevent any estimate of the second braking index, and render difficult measuring its first braking index. We assumed $m=3.5$, consistent with the low first braking index, $n=1.4 \pm 0.2$ (Lyne et al. 1996). As $n<2$, Vela has already passed its minimum $\dot{P}$ value and its predicted evolution is close to a $n=1$ line. We note that the track computed for the past evolution of Vela is close of that of PSR B0540-69. However, its motion in the $P-\dot{P}$ diagram is very slow and when integrating 


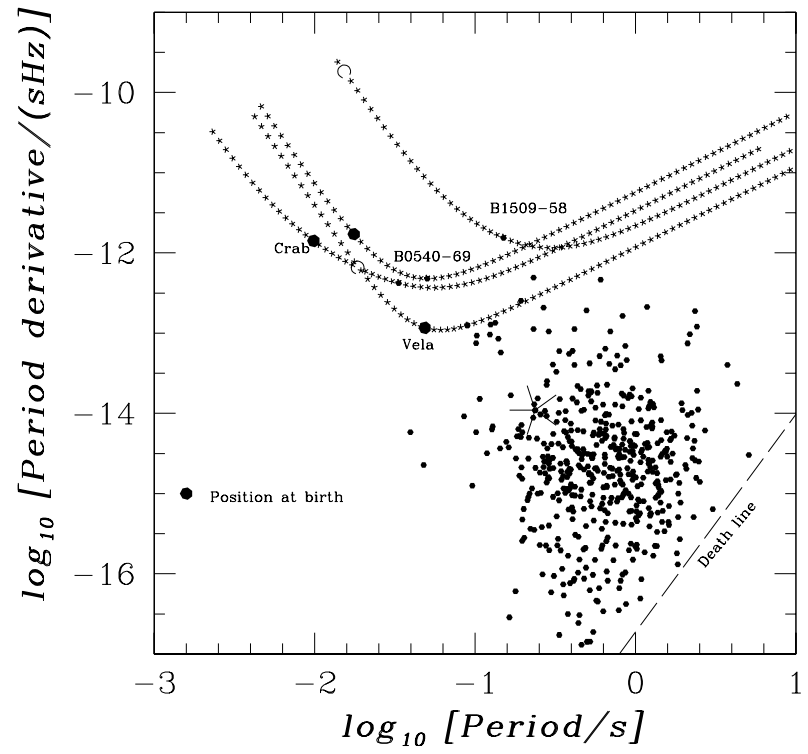

Fig. 3. Evolutionary trajectories computed for the Crab, PSR B1509-58, PSR B0540-69 and Vela. The small dots on the tracks mark the present positions of these four pulsars, while the big dots mark the positions integrated back one dynamical time. The open circle marks the position of the Crab at the time of the SN1054 explosion. For PSR B1509-58 the integration converges before one dynamical time, the large filled circle marking the $P-\dot{P}$ position with $t_{\mathrm{dyn}}=1$ year. The stationary model takes these pulsars into a region of the $P-\dot{P}$ diagram where pulsars have not been detected.

back one dynamical time Vela only reaches $P \approx 50 \mathrm{~ms}$ with a braking index almost equal to 2 .

Although exact tracks cannot be computed for older pulsars, their known timing parameters not constraining $\{g, r, s\}$ sufficiently, we evolved backwards 17 pulsars with $t_{\text {dyn }} \leq 10^{5}$ years, assuming $n=1.5$, in order to locate the region of their likely initial conditions in the $P-\dot{P}$ diagram (Fig. 5). We stopped the computation when $t_{\text {dyn }}=1$ year. We note that most of these pulsars arrive to higher $\dot{P}$ than all of the youngest pulsars, with the exception of PSR B1509-58. In order to get to $P-\dot{P}$ positions more consistent with that of the Crab, these pulsars would need to have a braking index closer to 1 . A prediction of the stationary multipole model is that $n \gtrsim 1$ for the majority of the pulsar population.

A more detailed discussion of the stationary model can be found in Alvarez (1998). Here we want to point out several inconsistencies of the model, which lead us to reject its non-time dependent version. As it can be seen in Fig. 3, the evolutionary tracks of the youngest pulsars take them to a region of the $P-\dot{P}$ diagram where no pulsars have been found. Although a large $\dot{P}$ might difficult the detection of pulsars located there, the region corresponds to pulsars slower but more energetic than some known radio-pulsars, specifically those with same $\dot{P}$ but lower $P$. Pulsars in that region should be detectable. We believe these tracks are indicative of the inconsistency between the model and the observations. A second discrepancy is the eventual dominance of the monopole term, which implies $v / \dot{v} \rightarrow$ constant (Fig. 6). As $n \rightarrow 1$ the dynamical time tends to a constant, $t_{\text {dyn }} \rightarrow 1 / 2 s$, which values between $2.8 \times 10^{3}$ years

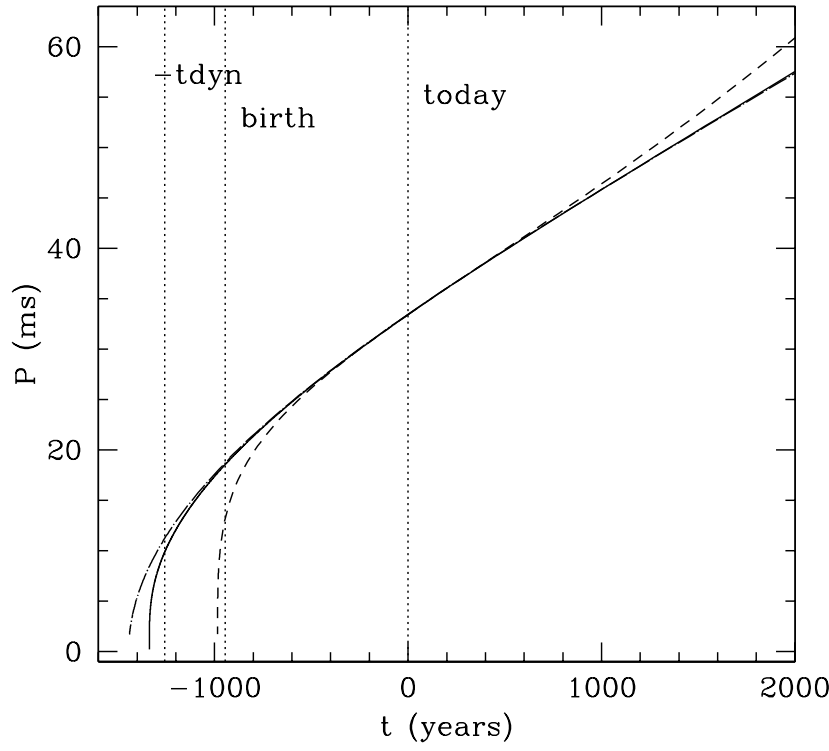

Fig. 4. Pulse period as function of time predicted for past and nearfuture evolution of the Crab pulsar. The evolutionary trajectories are given by the all allowed values of $m$. The dashed, full and dot-dashed lines correspond to $m=13.64, m=11.0$ and $m=10.82$ respectively. The vertical dotted lines mark the present time $(t=0)$, the moment of the SN1054 explosion ( $t=-948$ yrs $)$ and one dynamical age backwards.

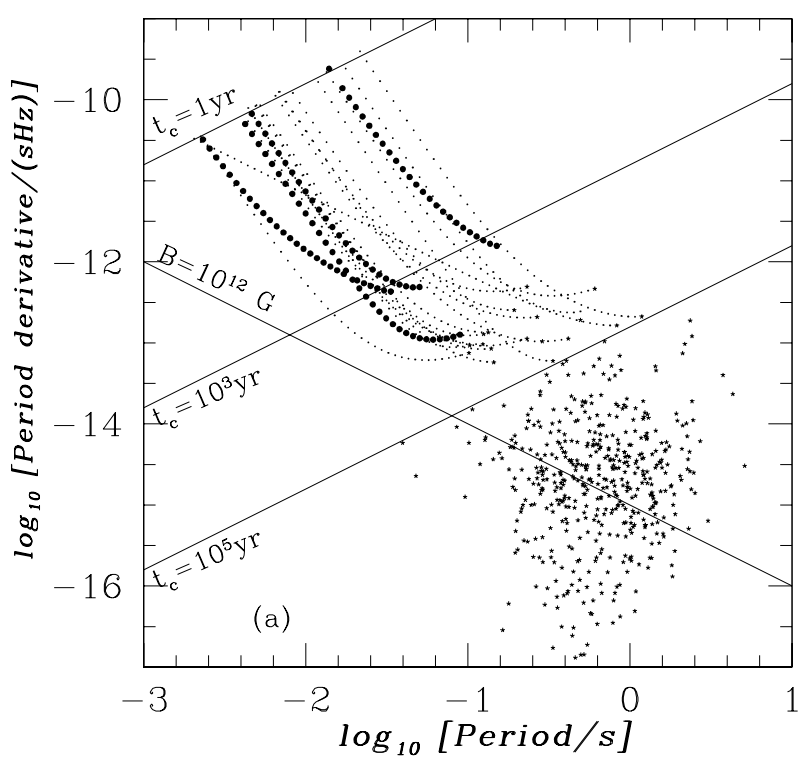

Fig. 5. Evolutionary trajectories for 17 middle aged pulsars evolved backwards to $t_{\mathrm{dyn}}=1$ year. The four youngest pulsars (Crab, PSR B1509-58, PSR B0540-69 and Vela) are denoted by the large dots.

for PSR B0540-69 and $13 \times 10^{3}$ years for Vela. The dynamical ages of the rest of the pulsars in the $P-\dot{P}$ diagram are much larger and cannot be reproduced by the model from the data of these young pulsars, a clear inconsistency between the predicted evolution of the young pulsars and the timing parameters of older pulsars.

Through the study of the stationary multipole model proposed here we reach the general conclusion that there is no time-independent evolutionary equation $\dot{v}=-f(v)$ able to 


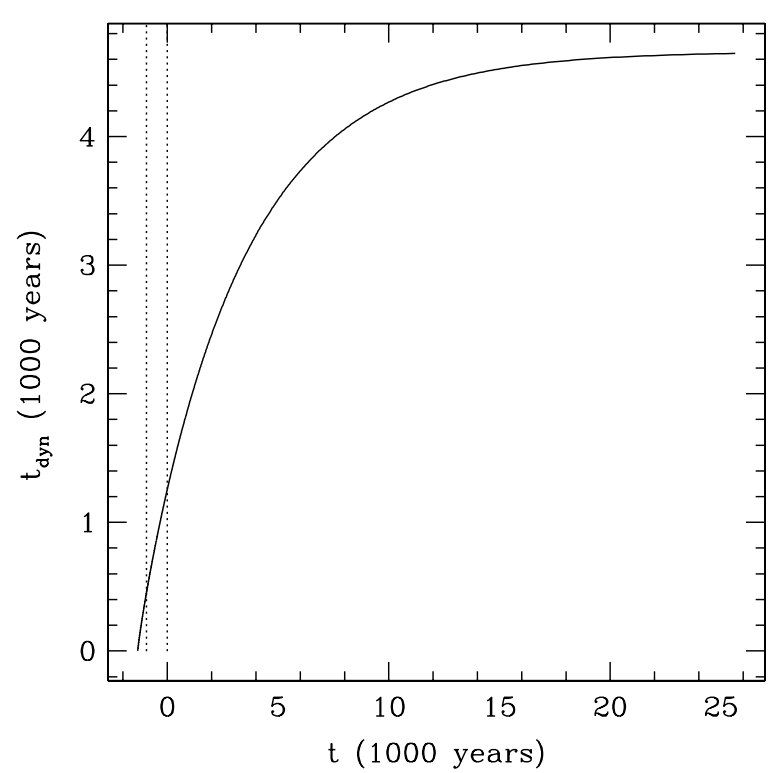

Fig. 6. Dynamical versus chronological time for the Crab pulsar. Note that $t_{\mathrm{dyn}} \rightarrow 4.6 \times 10^{3}$ yrs for ages greater than $\sim 20 \times 10^{3}$ years. As the model forces $\dot{v} \propto v$, as $n \rightarrow 1$, dynamical ages tend to constant values which, for the four pulsars studied, are below the values observed for other pulsars. The dotted lines mark the date of birth of the pulsar and today.

reconcile braking indices with the $P-\dot{P}$ diagram. Note that including a $\dot{v} \propto v^{2}$ term ${ }^{2}$ cannot prevent the eventual dominance of the monopolar term, needed to explain the braking index of Vela (and probably also of PSR B0540-69). The impossibility to have a time-independent expression means that the physical properties involved in the dynamics of pulsars must vary on timescales comparable to their life as active radio-pulsars. The most obvious form of time dependence is through the magnetic moment of the star, namely magnetic field decay or alignment betweenthe magnetic and rotational axes. We consider in the next section the time-dependent multipole model using physically expected time-dependences.

\section{The time-dependent multipole model}

Modelling the distribution observed in the $P-\dot{P}$ diagram requires considering plausible time dependences in physical parameters involved like the magnetic dipole moment of the neutron star. Observational evidence in favor of magnetic field decay is discussed by Tauris \& Konar (2001), who analyzed the relation between characteristic time against rotational energy of the pulsars in the Princeton Catalog (Taylor et al. 1993). Additional evidence comes from the measurements of their proper motions and from the study of ultramagnetized neutron stars or magnetars. Sang \& Chanmugam (1990) argued that dynamical and kinetic ages of pulsars can only be reconciliated with decaying magnetic fields of the form $B(t)=B(0) /\left(1+t / t_{\mathrm{c}}\right)$. Further evidence of magnetic field decay is provided by anomalous X-ray pulsars (AXPs) interpreted as magnetars. Colpi et al. (2000) required the magnetic field in these neutron star

\footnotetext{
${ }^{2}$ Inconsistent with the antisymmetric condition.
}

related objects to decay as a power law on timescales of the order of $10^{4}$ years.

We introduce time dependence in our model through the coefficients $\{r(t), s(t)\} \propto B(0)^{2} \psi\left(t / t_{\mathrm{c}}\right)$, where $t_{\mathrm{c}}$ is the characteristic time-scale for field decay and $\psi$ a dimensionless function satisfying $\psi(0)=1$. Frequently used functional forms of $\psi$ are exponential or inverse linear. For simplicity, we will only consider future evolution for the youngest pulsars, so that the quadrupolar coefficient $g(t)$ can be neglected hereafter ${ }^{3}$. We can then study evolutionary tracks on the $P-\dot{P}$ diagram writing Eq. (4) in terms of the period and its derivative as

$\dot{P}=\left(\frac{r_{0}}{P}+s_{0} P\right) \psi\left(t / t_{\mathrm{c}}\right)$.

This equation describes the period evolution of pulsars for a decaying magnetic field. It can be integrated analytically, leading to:

$P^{2}(t)=\left(r_{0} / s_{0}+P_{0}^{2}\right) \exp \left[2 s_{0} t_{\mathrm{c}} \Psi\left(t / t_{\mathrm{c}}\right)\right]-r_{0} / s_{0}$

where $P_{0} \equiv P(0)$ and $\Psi(x)=\int_{0}^{x} \psi(u) \mathrm{d} u$. If $\psi \rightarrow 0$ for $t \gg t_{\mathrm{c}}, \Psi$ will generally converge to a value $\Psi_{\infty}$ and the period will tend to a constant, $P_{\infty}$. This occurs because the spin-down mechanisms disappear as $\psi$ approaches zero. If one knows the present values of $\psi$ and $\dot{\psi}$, the initial parameters $r_{0}$ and $s_{0}$ can be derived from Eq. (7) and its time derivative:

$s_{0}=\frac{1}{2 \psi}\left(\frac{\dot{P}}{P}\right)\left(3-n+\frac{(-\dot{\psi}) / \psi}{\dot{P} / P}\right)$,
$r_{0}=\frac{P \dot{P}}{2 \psi}\left(n-1-\frac{(-\dot{\psi}) / \psi}{\dot{P} / P}\right)$,

where $n$ is the present braking index and a decay-law means $\dot{\psi}<0$. The decay timescale $t_{\mathrm{c}}$ is taken here as a free parameter to fit the data, to be determined by selecting physically meaningfull trajectories in the $P-\dot{P}$ diagram and $r_{0}>0$ and $s_{0}>0$. Note that $r_{0}$ and $s_{0}$ are both positive if

$n-1>\frac{(-\dot{\psi}) / \psi}{\dot{P} / P}>n-3$,

which relates $t_{\mathrm{c}}$ with the dynamical time and requires $n \geq 1$ always. On the other hand, old pulsars can now have arbitrarily large braking indices, as $n$ can go to $\infty$ when $\psi / \dot{\psi} \rightarrow$ const., $P \rightarrow P_{\infty}$ and $\dot{P} \rightarrow 0$. The largest loss of rotational energy occurs when $\dot{\psi} \rightarrow 0, \psi \rightarrow$ constant i.e. when $n$ is close to one.

In the next sections we present evolutionary tracks for the cases of exponential and linear magnetic field decay.

\subsection{Exponential decay}

Based on the observed deficit of pulsars with periods larger than 1 s, Ostriker \& Gunn (1969) proposed a rapid decrease of pulsar radio luminosity as result of exponentially decaying magnetic fields,

$B(t)=B_{0} \exp \left(-t / t_{\mathrm{c}}\right)$

\footnotetext{
${ }^{3}$ Neglecting $g$ also means that we do not require knowing $m$.
} 
Table 2. Time-dependent model parameters and exponential magnetic field decay timescales consistent with $r_{0} \geq 0$ and $s_{0} \geq 0$.

\begin{tabular}{lccccc}
\hline \hline Pulsar & $r_{0}$ & $s_{0}$ & \multicolumn{3}{c}{$t_{\mathrm{c}}(\mathrm{yrs})$} \\
& $\left(\mathrm{Hz}^{-1}\right)$ & $(\mathrm{Hz})$ & $\min$ & best & $\max$ \\
\hline Crab & $1.0 \times 10^{-14}$ & $4.0 \times 10^{-12}$ & 3300 & $4.5 \times 10^{4}$ & $8.0 \times 10^{4}$ \\
$1509-58$ & $2.1 \times 10^{-13}$ & $1.8 \times 10^{-12}$ & 3390 & $3.7 \times 10^{4}$ & $1.5 \times 10^{5}$ \\
0540-69 & $1.0 \times 10^{-14}$ & $6.8 \times 10^{-12}$ & 6600 & $2.5 \times 10^{4}$ & $5.0 \times 10^{4}$ \\
Vela & $2.3 \times 10^{-17}$ & $1.7 \times 10^{-12}$ & $1.1 \times 10^{5}$ & $\ldots$ & $2.0 \times 10^{5}$ \\
\hline
\end{tabular}

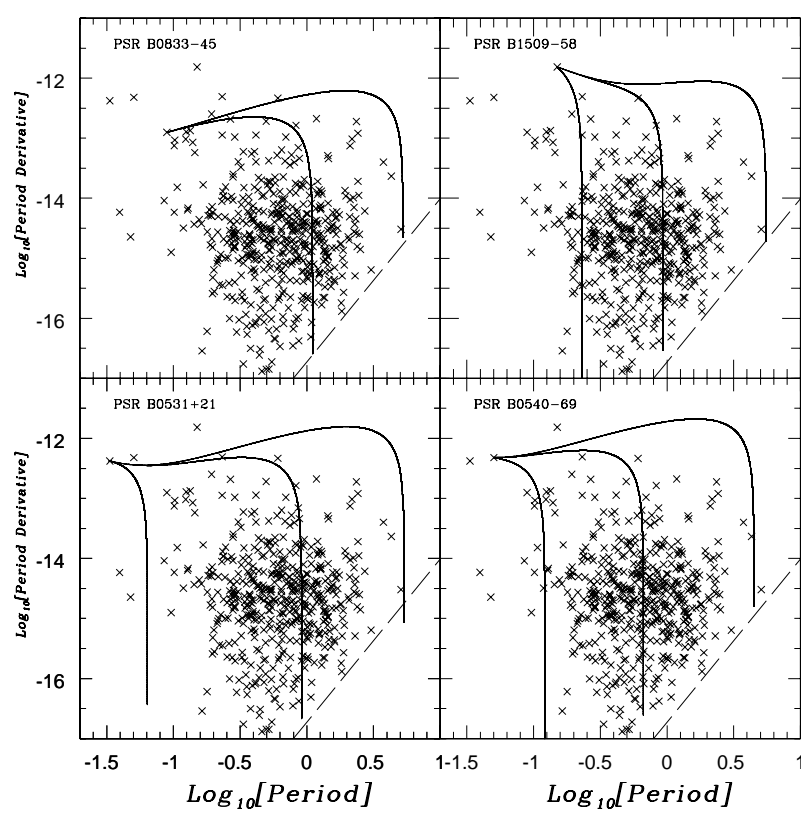

Fig. 7. Evolutionary trajectories using exponentially decaying magnetic fields for the Crab, PSR B1509-58, PSR B0540-69 and Vela. The three curves correspond to the lowest, best and largest $t_{\mathrm{c}}$ consistent with observations. For Vela the best trajectory is that of minimum $t_{\mathrm{c}}$, as shown in Table 2. Trajectories were followed for $10 t_{\mathrm{c}}$, $6 t_{\mathrm{c}}$ and $5 t_{\mathrm{c}}$ for the PSR B0531+21, 5.5 $t_{\mathrm{c}}$ and $4 t_{\mathrm{c}}$ for PSR B0833-45, $100 t_{\mathrm{c}}, 5.5 t_{\mathrm{c}}$ and $4 t_{\mathrm{c}}$ for PSR B1509-58 and $100 t_{\mathrm{c}}, 6 t_{\mathrm{c}}$ and $4.8 t_{\mathrm{c}}$ for PSR B0540-69.

a natural result of ohmic dissipation. In this case the model becomes

$\dot{P}=\mathrm{e}^{-2 t / t_{\mathrm{c}}}\left(r_{0} / P+s_{0} P\right)$,

and we have $\Psi_{\infty}=1 / 2$. For the youngest pulsars we obtain the trajectories shown in Fig. 7. Satisfactory trajectories are obtained for $t_{\mathrm{c}} \approx 10^{4} \mathrm{yrs}$ (Table 2 ), which correspond to extremely rapid evolution, as the magnetic field becomes negligible in just $10 t_{\mathrm{c}}$ and pulsars cross the death-line of the $P-\dot{P}$ diagram in $10^{4}$ to $10^{5}$ years. This timescales are too short to be consistent with estimated birth rates of one pulsar every 90 years (Brazier \& Johnston 1999) and supernova rates of one every 10-30 years (van der Bergh \& Tammann 1991). We conclude that exponentially decaying magnetic fields cannot be used to properly fit the $P-\dot{P}$ diagram without contradicting present known pulsar and supernova rates.
Table 3. Time-dependent model parameters and inverse linear magnetic field decay timescales consistent with $r_{0} \geq 0$ and $s_{0} \geq 0$.

\begin{tabular}{lccccc}
\hline \hline Pulsar & $\begin{array}{c}r_{0} \\
\left(\mathrm{~Hz}^{-1}\right)\end{array}$ & $\begin{array}{c}s_{0} \\
(\mathrm{~Hz})\end{array}$ & $\min$ & $\begin{array}{c}t_{\mathrm{c}} \text { (yrs) } \\
\text { best }\end{array}$ & $\max$ \\
\hline Crab & $9.4 \times 10^{-15}$ & $7.5 \times 10^{-12}$ & 2090 & $1.0 \times 10^{4}$ & $3.0 \times 10^{4}$ \\
$1509-58$ & $1.2 \times 10^{-13}$ & $4.8 \times 10^{-12}$ & 1830 & $1.0 \times 10^{4}$ & $4.0 \times 10^{4}$ \\
$0540-69$ & $7.3 \times 10^{-15}$ & $1.0 \times 10^{-11}$ & 4980 & $1.0 \times 10^{4}$ & $2.0 \times 10^{4}$ \\
Vela & $2.1 \times 10^{-18}$ & $1.7 \times 10^{-12}$ & $1.0 \times 10^{5}$ & $\ldots$ & $\ldots$ \\
\hline
\end{tabular}

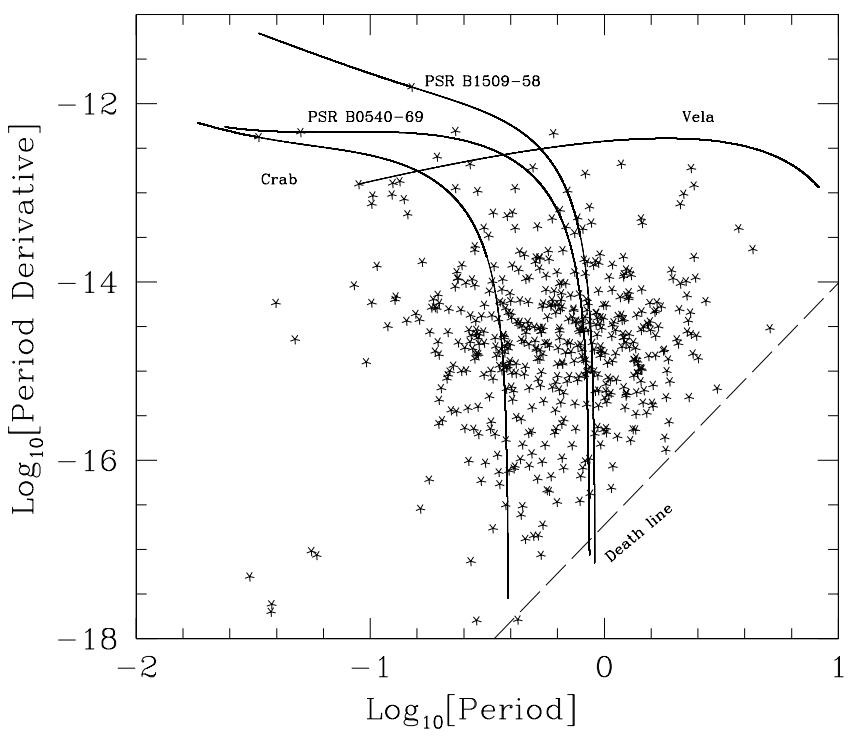

Fig. 8. Evolutionary trajectories for the Crab, PSR B1509-58, PSR B0540-69 and Vela, using an inverse linear decay-law for the magnetic field. The decay timescale used is $t_{\mathrm{c}}=10^{4}$ except for Vela where $t_{\mathrm{c}}=1.04 \times 10^{5}$ years. The trajectories were run for $10^{3} t_{\mathrm{c}}$, except that of Vela which was run for $10 t_{c}$.

\subsection{Inverse linear decay}

Sang \& Chanmugam (1990) proposed an inverse linear decay law for the magnetic field of pulsars,

$$
B(t)=\frac{B(0)}{1+t / t_{\mathrm{c}}}
$$

to reconcile dynamical and kinetic ages. Using this decay law in the model, relatively short timescales, $t_{\mathrm{c}} \sim 10^{4}$ years, are needed to fit the evolutionary trajectories of the four pulsars considered with the $P-\dot{P}$ distribution, as shown in Fig. 8 for the Crab, PSR B1509-58, PSR B0540-69 and Vela. Except for the Vela pulsar, to be discussed below, trajectories can be fitted through the bulk of the pulsar population. Although the timescales needed to fit the data are similar to those for the case of exponential decay, linear decay is far more convenient as pulsars remain active for $10^{3}-10^{4} t_{\mathrm{c}}$, crossing the death line of radio emission in some $10^{7}$ years, in reasonable agreement with pulsar and supernova rates.

For the Vela pulsar, the minimum decay timescales consistent with the model, i.e. with positive $r_{0}$ and $s_{0}$, do not manage to turn its evolutionary tracks towards the bulk of pulsars. However, the uncertainty in the measurement of $n$ allows us 


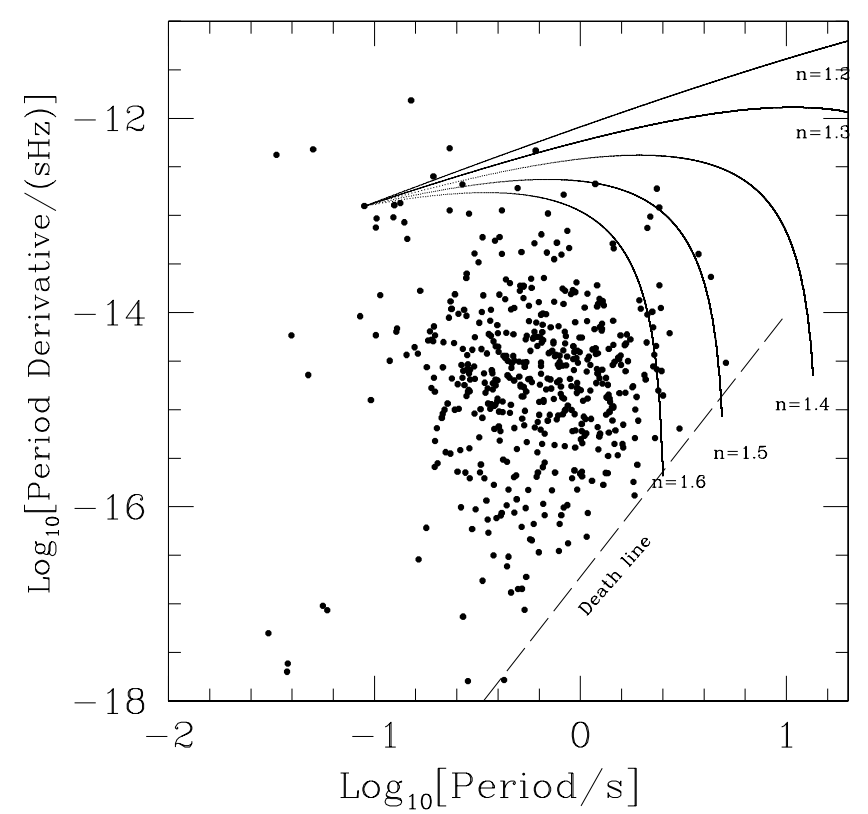

Fig. 9. Evolutionary trajectories for the Vela pulsar, considering different possible values of $n$. Trajectories using $n$ larger than $\sim 1.5$ are consistent with known long-period pulsars, while $n \leq 1.4$ is inconsistent with observations.

to consider a range of values. Evolutionary trajectories with $n \leq 1.4$ take Vela to a region of large periods and high period derivatives where no radio pulsars have been found. For $n=1.2$, Vela takes about $2.0 \times 10^{5}$ yrs to slow down to $P \sim 10 \mathrm{~s}$, and $3.6 \times 10^{5}$ years for $n=1.3$. Taking these numbers and considering one pulsar like Vela born every 11000 years, we should expect to see between 18 and 27 pulsars in the same region of the $P-\dot{P}$ diagram occupied by Vela. Indices $1.5 \leq n \leq 1.6$ can produce reasonable tracks (Fig. 9), leading to longer periods than those of the other three young pulsars, but consistent with long-period pulsars present in the $P-\dot{P}$ diagram. It does not seem feasible to reconcile Vela trajectories with those of the Crab, PSR B1509-58 and PSR B0540-69, which pass though the bulk of the pulsar population without impossing a braking index $n>1.6$, inconsistent (at least at the $1 \sigma$ level) with observations. So, even though the model can fit the data, the evidence points to the Vela pulsaras a particular type of pulsar. Pulsar evolution might distinguish between Crab-like pulsars, with magnetic-fields decaying at periods $P_{\infty} \lesssim 1 \mathrm{~s}$, and - less common - Vela-like pulsars, where the magnetic-field decay is slower and becomes spun-down to asymptotic periods between 1 and $10 \mathrm{~s}$.

\section{Physical spin-down mechanisms}

The main feature of the model presented here is the inclusion of the monopolar term, required to derive reasonable evolutionary tracks considering the measured braking indices. The other two terms of the model have been widely used in the past and are usually interpreted in terms of electromagnetic or gravitational radiation losses. An inclined rotating magnetic dipole in vacuum produces a dipolar term given by

$\dot{E}_{\text {dip }}=-\frac{2 \mu_{\perp}^{2}}{3 c^{3}} \Omega^{4}$,

where $\Omega=2 \pi \nu$ is the angular frequency and $\mu_{\perp}$ the component of magnetic dipole moment perpendicular to $\boldsymbol{\Omega}$. Pacini (1967) was the first to equate $\dot{E}_{\text {dip }}=I \Omega \dot{\Omega}$, introducing the basic equation of pulsar dynamics. A quadrupolar term can be associated either to the next electromagnetic moments or to the lowest gravitational radiation multipole moment:

$\dot{E}_{\text {quad }}=-\frac{32 G}{5 c^{5}} I^{2} e^{2} \Omega^{6}$,

where $I e$ the product of the moment of inertia and equatorial ellipticity of the star. The fact that observed indices are close to 3 indicates that gravitational radiation is not the dominant form of energy loss in pulsars at present, although it might be important in the very early stages of pulsar evolution.

Directly equating the Larmor equation to rotational losses cannot lead to the monopolar term proposed in Eq. (4). The Larmor equation arises from considering the energy flux of an electromagnetic wave, $\boldsymbol{S} \propto\left|\boldsymbol{E}_{\text {rad }}\right|^{2} \hat{k}$, with the radiation field $\boldsymbol{E}_{\text {rad }}$ proportional to the acceleration of the charged particles, scaling therefore as $\Omega^{n}$ with $n$ even and $\geq 4$. Even in the framework of the models mentioned in Sect. 2, it is unlikely that radiative emission processes can give a different dependence on $\Omega$.

On the other hand, there is clear evidence that particle acceleration and massive winds are important energy loss processes in pulsars. This evidence includes the short lifetimes of relativistic electrons in the Crab Nebula and the need of a source to account for its luminosity (as pointed out first by Oort \& Walraven 1956), observational changes directly observed in the vicinity of the Crab pulsar (Hester et al. 1995) and the X-ray images of the Crab, PSR B1509-58 and the Vela pulsar (Weisskopf et al. 2000; Gaensler et al. 2002; Helfand et al. 2001 respectively). The monopolar term cannot be of radiative origin, so we propose here its relation with particle and/or mass loss processes. We note we cannot discard a $\dot{E} \propto \Omega^{3}$ term but this term would be neither necessary nor sufficient to explain the data; therefore we avoid introducing it and assumed the antisymmetric condition for simplicity. Neglecting the $\dot{E} \propto \Omega^{3}$ term (equivalent to $\dot{v} \propto v^{2}$ ) we put forward the issue of whether particle acceleration and/or mass-loss follow an antisymmetric spin-down equation, like the Larmor equation does.

A monopolar spin-down term arises from any energy loss mechanism with a $\dot{E} \propto \Omega^{2}$ dependence, which can be expressed as

$\dot{E}_{\text {mon }}=-\beta\left(\frac{\mu_{\perp}^{2}}{c^{3}} \Omega^{4}\right)\left(\frac{c / \Omega}{R_{*}}\right)^{2}$,

where $\beta$ is a dimensionless factor that can depend on time but not explicitly on $\Omega$. It is an open question whether energy-loss processes like particle acceleration and pulsar winds can provide this sort of expression and we can only especulate about this point. Michel (1969) estimated the torque exerted on a neutron star by a pulsar wind as

$T=\left(\pi \Phi^{2} / 4 c\right) \Omega$ 
where $\Phi$ is the magnetic flux carried away by the wind. Assuming $\Phi$ can be constant, a pulsar wind would lead to a monopolar term in the energy loss equation. A detailed analysis of pulsar wind energetics has been given by Harding et al. (1999), who assumed an electric current flowing in the magnetosphere causing a magnetic torque on the star. The corresponding loss of rotational energy due to this torque is given by:

$\dot{E}_{\text {wind }}=\frac{c}{3} B_{*}^{2} R_{*}^{6}\left(\frac{1}{R_{\text {open }}^{2} R_{\mathrm{LC}}^{2}}\right)$,

where $R_{\mathrm{LC}}=c / \Omega . R_{\text {open }}$ is the distance from the center of the star to the magnetosphere region at which the magnetic field lines become open due to the pulsar wind. For $R_{\text {open }}$ independent of $\Omega$, this implies $\dot{E}_{\text {wind }} \propto \Omega^{2}$, i.e., a monopolar-like term.

\section{Conclusions}

A multipole equation for pulsar evolution can be derived naturally as the Taylor expansion of the general equation $\dot{v}=$ $-f(v, t)$. While it might give insight into short term evolution of pulsars, the time independent version of the multipole equation is inconsistent with observations, indicating that no equation of the form $\dot{v}=-f(v)$ is able to fully model pulsar spindown evolution. A time dependent multipole equation considering exponentially decaying magnetic-fields cannot reproduce the data without shortening the active life of pulsars below $10^{5}$ years. Introducing an inverse linear magnetic field decay with timescales of the order of $10^{4}$ years gives good agreement with observations and allows pulsars to remain active about $10^{7}$ years, in agreement with pulsar and supernova birth rates. The model is consistent with observations of the Vela pulsar only if its braking index is $\gtrsim 1.5$. The different trajectories of this pulsar relative to those of the Crab, PSR B1509-58 and PSR B0540-69 suggest the existence two types of pulsars: those with relatively short decay timescales which evolve to periods $P_{\infty} \lesssim 1 \mathrm{~s}$ and Vela-like pulsars, with more persistent magnetic fields evolving to the larger period pulsars observed in the $P-\dot{P}$ diagram. We believe the need to introduce a monopolar term in the spin-down equation is an indirect observational evidence that particle acceleration and/or mass-loss processes must follow $\dot{E} \propto \Omega^{2}$.

Acknowledgements. César Alvarez thanks Dany Page for his valuable comments during the preparation of his Ph.-D. Thesis. César Alvarez also thanks CONACyT for the $\mathrm{Ph}$. D. support grant received (reference number 86472).

\section{References}

Alvarez, C., \& Carramiñana, A. 1998, RMxAC, 7, 207

Alvarez, C. 2002, Ph.D. Thesis, INAOE

Blandford, R. D., \& Romani, R. W. 1988, MNRAS, 234, 57

Brazier, K. T. S., \& Johnston, S. 1999, MNRAS, 305, 671

Camilo, F. 1996, Pulsars: Problems and progress, ed. S. Johnston, M. A. Walker, \& M. Bailes, ASP Conf. Ser., 105, 39

Camilo, F., Kaspi, V. M., \& Lyne, A. G. 2000, ApJ, 541, 367

Chanmugam, G., \& Sang, Y. 1989, MNRAS, 241, 295

Colpi, M., Geppert, U., \& Page, D. 2000, ApJ, 529, L29

Chubarian, E., Grigorian, H., Poghosyan, G., \& Blaschke, D. 2000, A\&A, 357, 968

Ferrari, A., \& Ruffini, R. 1969, ApJ, 158, L71

Gaensler, B. M., Arons, J., Kaspi, V. M., et al. 2002, ApJ, 569, 878

Glendenning, N. K. 1996, Compact Stars (New York: SpringerVerlag)

Glendenning, N. K., Pei, S., \& Weber, F. 1997, Phys. Rev. Lett., 79, 1603

Gold, T. 1968, Nature, 218, 731

Goldreich, P. 1970, ApJ, 160, L11

Harding, A. K., Contopoulos, I., \& Kazanas, D. 1999, ApJ, 525, L125

Helfand, D. J., Gotthelf, E. V., \& Halpern, J. P. 2001, ApJ, 556, 380

Hester, J. J., Scowen, P. A., Sankrit, R., et al. 1995, ApJ, 448, 240

Kaspi, V. M., Manchester, R. N., Siegman, B., Johnston, S., \& Lyne, A. G. 1994, ApJ, 422, L83

Lyne, A. G., Pritchard, R. S., \& Smith, F. G. 1988, MNRAS, 233, 667

Lyne, A. G., Pritchard, R. S., Graham-Smith, F., \& Camilo, F. 1996, Nature, 381, 497

Manchester, R. N., \& Taylor, J. H. 1977, Pulsars (San Francisco: Freeman)

Manchester, R. N., Newton, L. M., \& Durdin, J. M. 1985, Nature, 313, 374

Manchester, R. N., \& Peterson, B. A. 1989, ApJ, 342, L23

Mereghetti, S., Bandiera, R., Bocchino, F., \& Israel, G. L. 2002, ApJ, 574,873

Michel, C. F. 1969, ApJ, 158, 727

Oort, J. H., \& Walraven, Th. 1956, Bull. Astron. Inst. Neth., 12, 285

Ostriker, J. P., \& Gunn, J. E. 1969, ApJ, 157, 1395

Pacini, F. 1967, Nature, 216, 567

Pacini, F. 1968, Nature, 219, 145

Sang, Y., \& Chanmugam, G. 1990, ApJ, 363, 597

Taylor, J. H., Manchester, R. N., \& Lyne, A. G. 1993, ApJS, 88, 529

Tauris, T. M., \& Konar, S. 2001, A\&A, 376, 543

van der Bergh, S., \& Tammann, G. A. 1991, ARA\&A, 29, 363

Weisskopf, M. C., Hester, J. J., Tennant, A. F., et al. 2000, ApJ, 536, L81 